\title{
Site-resolved and quantitative characterization of very weak protein-ligand interactions
}

\author{
Brian Fuglestad $^{\dagger}$, Nicole E. Kerstetter ${ }^{\ddagger}$, A. Joshua Wand $^{\dagger, \ddagger},{ }^{*}$ \\ †Johnson Research Foundation and Department of Biochemistry \& Biophysics, Perelman School \\ of Medicine at the University of Pennsylvania, Philadelphia, PA, United States \\ FGraduate Group in Biochemistry \& Molecular Biophysics, Perelman School of Medicine at the \\ University of Pennsylvania, Philadelphia, PA, United States
}

\section{Abstract}

\begin{abstract}
Very weak interactions between small organic molecules and proteins have long been predicted and are expected to have dissociation constants of 100s of $\mathrm{mM}$ and above. Unfortunately, quantitative evaluation of binding in a high-resolution structural context for this affinity regime is particularly difficult and often impossible using existing experimental approaches. Here we show that nanoscale encapsulation of single protein molecules within the water core of reverse micelles enables the detection and evaluation of weak binding interactions at atomic resolution using solution NMR spectroscopy. This strategy is used to survey the interactions of a set of small molecules with the cytokine interleukin- $1 \beta$ (IL- $1 \beta$ ). The interaction of IL- $1 \beta$ with these molecules is found to vary from more diffuse and weak binding modes to more specific and with a relatively higher affinity. The interactions detected here cover a large portion of the protein surface and have dissociation constants mostly in the low molar-range. These results illustrate the ability of a protein to interact productively with a variety of small molecule functional groups and point to a broader potential to target even relatively featureless proteins surfaces for applications in chemical biology and drug discovery.
\end{abstract}

\begin{abstract}
Proteins are suspected of being rich in sites that bind weakly to small-molecules. These "hotspots" are thought to involve a variety of surface features and not limited to natural ligand binding sites. ${ }^{1-3}$ The potential utility of weak protein-small molecule interactions lies in their potential to strengthen the robustness of the design and development of small molecule drugs and tools for chemical biology. However, the direct experimental detection and characterization of weak protein complexes with small-molecules is generally difficult and is rarely pursued. On the other hand, a number of computational approaches are being developed to predict weak interactions between very small molecules to proteins, ${ }^{4}$ but progress has been hampered by limited experimental benchmarks.
\end{abstract}

\footnotetext{
"Corresponding Author: A. Joshua Wand. wand@pennmedicine.upenn.edu. Supporting Information Available: This material is available free of charge via the Internet. Details of the experimental methods, supplementary figures 1-4, supplementary tables 1-3, supplemental references (PDF) The authors declare no competing financial interests.
} 
Experimental detection of weak interactions of very small organic molecules with proteins finds its roots in a handful of crystallographic studies. ${ }^{2,3,5-7}$ Crystallographic detection is limited to those proteins for which the large concentrations of ligand required to detect binding do not disrupt the crystals. ${ }^{3}$ While cross-linking proteins helps suppress crystal disruption, ${ }^{5}$ few protein systems are apparently amenable to this approach although it is noted that slightly larger "mini-fragments" (MW 90 Da) are apparently less problematic. ${ }^{8}$ Regardless, only qualitative characterization of weak interactions is available through a crystallographic analysis since affinity cannot be measured.

In principle, solution NMR is well poised for the detection and characterization of proteinsmall molecule interactions but has only seen limited use in this context. ${ }^{9,10}$ The large concentration of small molecule required to form extremely weak $\left(\mathrm{K}_{\mathrm{d}}>100 \mathrm{mM}\right)$ interactions has a number deleterious effects including degradation of NMR probe performance through dielectric loss, introduction of overwhelming spectral artifacts or signals, and destabilization and unfolding of the protein.

Here we explore the capabilities of reverse micelle (RM) encapsulation to evade the issues that thwart the use of solution NMR to characterize very weak protein-ligand interactions. Under the conditions optimal for solution NMR, the reverse micelle is composed of a water core contained within a nearly spherical shell of surfactant molecules with their polar head groups pointed inwards and their hydrophobic tails pointed outwards. Reverse micelles optimal for NMR (i.e. small \& nearly spherical) are created under water-limited conditions. ${ }^{11}$ Under a range of compositions of surfactant, water, and alkane solvent, the population of reverse micelles is remarkably uniform in its physical attributes. ${ }^{12,13}$ The uniformity and high quality of NMR spectra combined with other chemical characteristics also demonstrate that reverse micelles are constantly exchanging their contents on times scales much faster than $10^{3}$ per second. ${ }^{11}$ Indeed, previous experiments have confirmed very fast exchange of RM contents. ${ }^{11,14-16}$ In essence, rapid exchange ensures that contents of the RM aqueous cores are in equilibrium and behave as one single phase. These features ensure that bulk properties, such as $\mathrm{pH}$, and the meaning and integrity of binding phenomena are preserved. Reverse micelle surfactant systems have been developed to encapsulate proteins with high structural fidelity. ${ }^{17}$ Particularly important here is the LDAO/10MAG surfactant system, which is able to robustly and universally encapsulate proteins. ${ }^{17,18}$

Under the conditions utilized in this study, the aqueous phase of a RM system represents $\sim 2 \%$ of the sample volume. The small fractional volume provides two important advantages: it allows greatly reduced amount of material to be used to reach equivalent concentrations in the RM core (Supplementary Figure 1) and it eliminates many of the spectral artifacts that limit NMR spectroscopy of weak interactions between small molecules and proteins using bulk solution samples. For example, solutions of RM encapsulated protein molecules in high ionic aqueous solution have an effective low conductivity, which preserves the full performance of the NMR probe. ${ }^{19}$ Similarly, high effective concentrations of ligand in the $\mathrm{RM}$ aqueous core represent low overall concentrations to the NMR spectrometer allowing standard phase cycling and gradient selection strategies to eliminate unwanted signals that generally overwhelm similar strategies in bulk solution experiments. ${ }^{11,17}$ With respect to the sample itself, the addition of large quantities of polar small molecules can often significantly 
alter the solution $\mathrm{pH}$. The zwitterionic LDAO has a $\mathrm{pKa} \sim 5$ and an effective concentration of $1.5 \mathrm{M}$ within the RM core, providing significant buffering capacity. ${ }^{16}$ The high buffering capacity largely eliminates confounding $\mathrm{pH}$ effects on the protein NMR spectrum. The confined space of the RM can also stabilize the protein from denaturing effects. ${ }^{20-22}$ This is especially important when considering probe molecules such as acetonitrile and alcohols that are known protein denaturants. ${ }^{23,24}$

To explore the potential of RM NMR to characterize weak protein-ligand interactions we have chosen a set of primitive small molecules used in the "cosolvent" molecular dynamics approach termed MixMD. ${ }^{4}$ These were pyrimidine, methylammonium, acetonitrile, ethanol, $\mathrm{N}$-methylacetamide, and imidazole. Interleukin- $1 \beta$ (IL-1 $\beta$ ) was used as a test protein. IL-1 $\beta$ is an important drug target for inflammatory and other diseases ${ }^{25}$ It has relatively featureless surface, which is a common characteristic of signaling proteins that engage in protein-protein interactions. Drugging efforts aimed at IL- $1 \beta$ or other 'featureless' proteins would involve binding a small molecule to a protein-protein interfacing contact surface to disrupt the receptor complex, rather than a ligand-binding pocket. ${ }^{26}$

All cosolvents benefited in some way from using RM encapsulation. The ionic character of methylammonium chloride and imidazole conspire to degrade the performance of the cryogenically cooled NMR probe such that concentrations must be restricted to $<400 \mathrm{mM}$ in bulk experiments. Ethanol and acetonitrile give rise to significant artifacts from the aliphatic proton resonances, although they do not interfere with the amide spectrum. Deuteration of cosolvent could be used to eliminate this issue but is unnecessary when RM encapsulation is used. $\mathrm{N}$-methylacetamide displays a sharp resonance corresponding to natural abundance ${ }^{15} \mathrm{~N}$, which at high concentrations obscures one resonance in the IL-1 $\beta$ spectrum. Pyrimidine at high concentrations results in severe artifacts from the amino protons in bulk solution samples (Supplementary Figure 2). Reverse micelle encapsulation permits use of high effective concentrations of all cosolvents by avoiding these artifacts.

A potential limitation of using RMs for experimental cosolvent mapping is the partitioning of the cosolvent into the bulk alkane phase. It is noted that most published partitioning experiments and established $\log \mathrm{P}$ calculators are based on the octanol/water solvent system. ${ }^{27}$ Using a simple NMR method ${ }^{28}$ modified for alkane/water partitioning, the $\log \mathrm{P}$ values of the cosolvents in alkane/water were measured (Supplementary Table 1). As would be expected from earlier comparisons, ${ }^{27}$ the partition coefficients of the cosolvent molecules referencing pentane are shifted to more negative values than the classic octanol/water system (Supplementary Table 1). As a result, even for the more positive octanol/water partitioning values (acetonitrile, pyrimidine), over one-third of the molecules are retained in the RM and maintain the advantage of the RM approach over measurements in bulk water. The measured partition coefficients were used to calculate the concentration of cosolvent in the RM core.

The cosolvent molecules were individually titrated into solutions of IL-1 $\beta$ encapsulated in LDAO/10MAG reverse micelles prepared in pentane. IL- $1 \beta$ was concentrated to $5 \mathrm{mM}$ and delivered to the RM system via direct injection of the aqueous phase. ${ }^{17}$ This ensures that the effective concentration of protein within the aqueous phase of the RM system is well controlled. Samples remained visually clear through all titrations. 
Chemical shift perturbations (CSPs) were used to identify specific binding. Specific binding, as opposed to bulk solvent effects, is characterized by site saturation, CSPs that are spatially localized in the protein structure and can be described by a single dissociation constant. High quality saturation curves were obtained and allowed precise determination of binding beyond even 5 M (Supplementary Figure 3 and Supplementary Table 2). Broad, linear shits due to solvent effects were not observed. Saturation curves all clearly reflected a two-state equilibrium. The differing binding patterns of each cosolvent to the IL- $1 \beta$ surface eliminates the possibility that simply changing bulk solvent properties by cosolvent addition perturbs the protein. Comparison of cosolvent titration data with the $\mathrm{pH}$ dependence of spectra of RM encapsulated IL- $1 \beta^{17}$ indicate that the $\mathrm{pH}$ was unchanged throughout the titrations. As might be expected for binding involving a limited number of primitive interactions, we find that the surface of IL- $1 \beta$ is littered with weak binding sites (Figure 1).

To define a binding site, groups of nearby residues showing a CSP of its amide ${ }^{15} \mathrm{~N}-{ }^{1} \mathrm{H}$ correlation were spatially clustered using a k-means algorithm and the binding curves fitted globally to a single dissociation constant (Supplementary Table 3). Individual cosolvents generally display multiple distinct and well-defined binding sites on the surface of the protein (Figure 1). Conversely, many regions of the protein surface bind several of the cosolvents and are effectively "hot spots" capable of interacting with a range of chemical moieties (Figure 2A).

Many of the residues that interact with 3 or more cosolvents are localized to the surface of IL-1 $\beta$ that engages its receptor binding partners (Figure 2B). ${ }^{29}$ This reflects a previous solvent mapping crystallography/computational study of Ras. ${ }^{30}$ With exception of the GTP binding site, Ras is also relatively featureless and engages in protein-protein interactions. Like the IL- $1 \beta$ results reported here, Ras displayed a propensity to bind cosolvents in protein binding surfaces. Together these results suggest that cosolvent interactions of this type may be useful for revealing druggable hotspots and my aid in design of inhibitors of proteinprotein interfaces.

The distribution of dissociation constants arising from these weak but specific binding interactions is striking. Fitted $\mathrm{K}_{\mathrm{d}}$ values ranged from $100 \mathrm{~s}$ of $\mathrm{mM}$ to $5 \mathrm{M}$ and are centered around $1.5-2.5 \mathrm{M}$ (Figure 2C). Acetonitrile and $\mathrm{N}$-methylacetamide show relatively sparse binding across the protein surface (Figure 1E and 1D) and display relatively weak binding affinities (Figure 2D). Imidazole, pyrimidine and, to a lesser extent, methylammonium, bind at a larger number of sites (Figure 1). Interestingly, nitrogen containing heterocycles such as imidazole and pyrimidine are common drug and inhibitor scaffolds and their propensity to bind to many protein sites may be advantageous. ${ }^{31,32}$ Ethanol is somewhat unique in that its binding is limited to fewer sites (Figure 1C) and, with some exceptions, displays relatively high binding affinity within a narrow $\mathrm{K}_{\mathrm{d}}$ range (Figure 2D).

Ethanol was the only cosolvent that could also be successfully titrated under standard bulk conditions. Comparison of the dissociation constants obtained in bulk and RM titrations gave a reasonable correlation $\left(R^{2}=0.80, R M S D=0.87 \mathrm{M}\right.$, Supplementary Figure $\left.4 \mathrm{~A}\right)$. The slope of the correlation plot is 0.60 indicating that ethanol binds slightly more weakly to the protein in RMs than in bulk. This is possibly due to reduced dynamics of water in the 
reverse micelle leading to a reduced solvent entropy gain upon displacement of protein surface water. ${ }^{33-36}$ Nevertheless, the surface mapping is accurate as demonstrated by comparing $\mathrm{CSP}_{\max }$ values derived in bulk aqueous and RM measurements (slope of 1.05; $\mathrm{R}^{2}$ $=0.77$; Supplementary Figure 4B).

In summary, a method has been presented that leverages advantages of RM based protein NMR to measure small-molecule interactions with proteins in the extremely low affinity regime that has been largely inaccessible. The resulting cosolvent mapping of IL- $1 \beta$ reveals a variety of binding modes of small-molecules to a protein lacking a ligand-binding pocket: from more diffuse and weak (i.e. acetonitrile and $\mathrm{N}$-methylacetamide), to more specific and with a relatively higher affinity (i.e. ethanol and imidazole). These results potentially provide an experimental benchmark for molecular dynamics simulations that strive to identify and characterize these interactions. The RM approach promises to enable a wide range of protein systems to be mapped for small-molecule interaction sites and druggable hot spots.

\section{Supplementary Material}

Refer to Web version on PubMed Central for supplementary material.

\section{ACKNOWLEDGMENT}

We gratefully acknowledge K. Valentine and K. Mitchell-Koch for helpful discussion. This work was supported by NIH grant R21 CA206958 to A. J. W.

\section{REFERENCES}

(1). Bauman JD, Patel D, Dharia C, Fromer MW, Ahmed S, Frenkel Y, Vijayan RS, Eck JT, Ho WC, Das K, Shatkin AJ, and Arnold E (2013) Detecting allosteric sites of HIV-1 reverse transcriptase by X-ray crystallographic fragment screening, J. Med. Chem 56, 2738-2746. [PubMed: 23342998]

(2). Mattos C, Bellamacina CR, Peisach E, Pereira A, Vitkup D, Petsko GA, and Ringe D (2006) Multiple solvent crystal structures: probing binding sites, plasticity and hydration, J. Mol. Biol 357, 1471-1482. [PubMed: 16488429]

(3). Mattos C, and Ringe D (1996) Locating and characterizing binding sites on proteins, Nature Biotechnol. 14, 595. [PubMed: 9630949]

(4). Ghanakota P, and Carlson HA (2016) Driving Structure-Based Drug Discovery through Cosolvent Molecular Dynamics, J. Med. Chem 59, 10383-10399. [PubMed: 27486927]

(5). Allen KN, Bellamacina CR, Ding X, Jeffery CJ, Mattos C, Petsko GA, and Ringe D (1996) An experimental approach to mapping the binding surfaces of crystalline proteins, J. Phys. Chem 100, 2605-2611.

(6). English AC, Done SH, Caves LS, Groom CR, and Hubbard RE (1999) Locating interaction sites on proteins: the crystal structure of thermolysin soaked in $2 \%$ to $100 \%$ isopropanol, Proteins 37 , 628-640. [PubMed: 10651278]

(7). Dechene M, Wink G, Smith M, Swartz P, and Mattos C (2009) Multiple solvent crystal structures of ribonuclease A: an assessment of the method, Proteins 76, 861-881. [PubMed: 19291738]

(8). O'Reilly M, Cleasby A, Davies T, Hall R, Ludlow RF, Murray C, Tisi D, and Jhoti H (2019) Crystallographic screening using ultra-low-molecular-weight ligands to guide drug design, Drug Discov. Today 24, 1081-1086. [PubMed: 30878562]

(9). Toyama Y, Kano H, Mase Y, Yokogawa M, Osawa M, and Shimada I (2018) Structural basis for the ethanol action on G-protein-activated inwardly rectifying potassium channel 1 revealed by NMR spectroscopy, Proc. Natl. Acad. Sci. U. S. A 115, 3858-3863. [PubMed: 29581303] 
(10). Liepinsh E, and Otting G (1997) Organic solvents identify specific ligand binding sites on protein surfaces, Nature Biotechnol 15, 264-268. [PubMed: 9062927]

(11). Nucci NV, Valentine KG, and Wand AJ (2014) High-resolution NMR spectroscopy of encapsulated proteins dissolved in low-viscosity fluids, J. Magn. Reson 241, 137-147. [PubMed: 24656086]

(12). Fuglestad B, Gupta K, Wand AJ, and Sharp KA (2016) Characterization of cetyltrimethylammonium bromide/hexanol reverse micelles by experimentally benchmarked molecular dynamics simulations., Langmuir 32, 1674-1684. [PubMed: 26840651]

(13). Fuglestad B, Gupta K, Wand AJ, and Sharp KA (2019) Water loading driven size, shape, and composition of cetyltrimethylammonium /hexanol/pentane reverse micelles, J. Colloid Interface Sci 540, 207-217. [PubMed: 30640068]

(14). Carlstrom G, and Halle B (1988) Nuclear-spin quenching - A new probe of exchange kinetics and droplet size in disperse systems., Mol. Phys 64, 659-678.

(15). Carlstrom G, and Halle B (1989) Shape fluctuations and water diffusion in microemulsion droplets - A nuclear-spin relaxatoin study., J. Phys. Chem 93, 3287-3299.

(16). Marques BS, Nucci NV, Dodevski I, Wang KW, Athanasoula EA, Jorge C, and Wand AJ (2014) Measurement and control of $\mathrm{pH}$ in the aqueous interior of reverse micelles, J. Phys. Chem. B 118, 2020-2031. [PubMed: 24506449]

(17). Fuglestad B, Marques BS, Jorge C, Kerstetter NE, Valentine KG, and Wand AJ (2019) Reverse Micelle Encapsulation of Proteins for NMR Spectroscopy, Methods Enzymol. 615, 43-75. [PubMed: 30638537]

(18). Dodevski I, Nucci NV, Valentine KG, Sidhu GK, O’Brien ES, Pardi A, and Wand AJ (2014) Optimized reverse micelle surfactant system for high-resolution NMR spectroscopy of encapsulated proteins and nucleic acids dissolved in low viscosity fluids, J. Am. Chem. Soc 136, 3465-3474. [PubMed: 24495164]

(19). Flynn PF, Mattiello DL, Hill HD, and Wand AJ (2000) Optimal use of cryogenic probe technology in NMR studies of proteins, J. Am. Chem. Soc 122, 4823-4824.

(20). O'Brien ES, Nucci NV, Fuglestad B, Tommos C, and Wand AJ (2015) Defining the Apoptotic Trigger: The interaction of cytochrome c and cardiolipin, J. Biol. Chem 290, 30879-30887. [PubMed: 26487716]

(21). Peterson RW, Anbalagan K, Tommos C, and Wand AJ (2004) Forced folding and structural analysis of metastable proteins, J. Am. Chem. Soc 126, 9498-9499. [PubMed: 15291527]

(22). Nucci NV, Fuglestad B, Athanasoula EA, and Wand AJ (2014) Role of cavities and hydration in the pressure unfolding of T4 lysozyme, Proc. Natl. Acad. Sci. U. S. A 111, 13846-13851. [PubMed: 25201963]

(23). Herskovits TT, Gadegbeku B, and Jaillet H (1970) On the structural stability and solvent denaturation of proteins I. Denaturation by the alcohols and glycols, J. Biol. Chem 245, 25882598. [PubMed: 5445802]

(24). Griebenow K, and Klibanov AM (1996) On protein denaturation in aqueous-organic mixtures but not in pure organic solvents, J. Am. Chem. Soc 118, 11695-11700.

(25). Dinarello CA (2011) Interleukin-1 in the pathogenesis and treatment of inflammatory diseases, Blood 117, 3720-3732. [PubMed: 21304099]

(26). Wells JA, and McClendon CL (2007) Reaching for high-hanging fruit in drug discovery at protein-protein interfaces, Nature 450, 1001-1009. [PubMed: 18075579]

(27). Leo A, Hansch C, and Elkins D (1971) Partition coefficients and their uses, Chem. Rev 71, 525616.

(28). Cumming H, and Ruücker C. (2017) Octanol-Water Partition Coefficient Measurement by a Simple 1H NMR Method, ACS Omega 2, 6244-6249. [PubMed: 31457869]

(29). Thomas C, Bazan JF, and Garcia KC (2012) Structure of the activating IL-1 receptor signaling complex, Nat. Struct. Mol. Biol 19, 455. [PubMed: 22426547]

(30). Buhrman G, Casey O, Zerbe B, Kearney BM, Napoleon R, Kovrigina EA, Vajda S, Kozakov D, Kovrigin EL, and Mattos C (2011) Analysis of binding site hot spots on the surface of Ras GTPase, J. Mol. Biol 413, 773-789. [PubMed: 21945529] 
(31). DeSimone R, Currie K, Mitchell S, Darrow J, and Pippin D (2004) Privileged structures: applications in drug discovery, Comb. Chem. High Throughput Screen 7, 473-493. [PubMed: 15320713]

(32). Welsch ME, Snyder SA, and Stockwell BR (2010) Privileged scaffolds for library design and drug discovery, Curr. Opin. Chem. Biol 14, 347-361. [PubMed: 20303320]

(33). Nucci NV, Pometun MS, and Wand AJ (2011) Mapping the hydration dynamics of ubiquitin, J. Am. Chem. Soc 133, 12326-12329. [PubMed: 21761828]

(34). Dahanayake JN, and Mitchell-Koch KR (2018) Entropy connects water structure and dynamics in protein hydration layer, Phys. Chem. Chem. Phys 20, 14765-14777. [PubMed: 29780979]

(35). Dzugutov M (1996) A universal scaling law for atomic diffusion in condensed matter, Nature 381, 137-139.

(36). Rosenfeld Y (1977) Relation between transport-coefficients and internal entropy of simple systems, Phys. Rev. A 15, 2545-2549. 
A

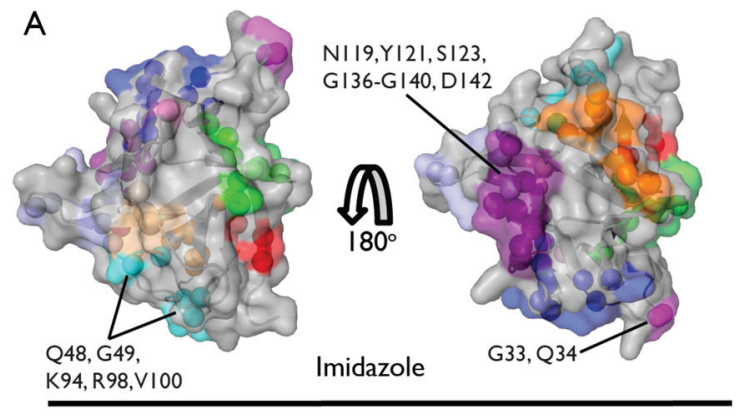

C

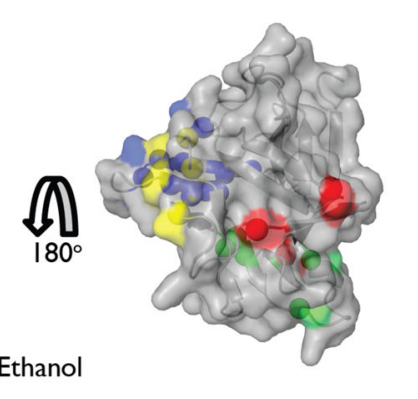

Y68, S70,V72-K74
T79-E83, QII6

E

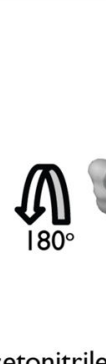

Figure 1. methylammonium chloride.
B

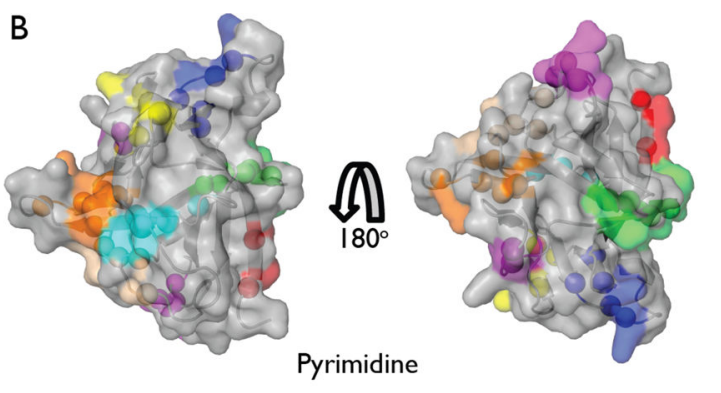

D

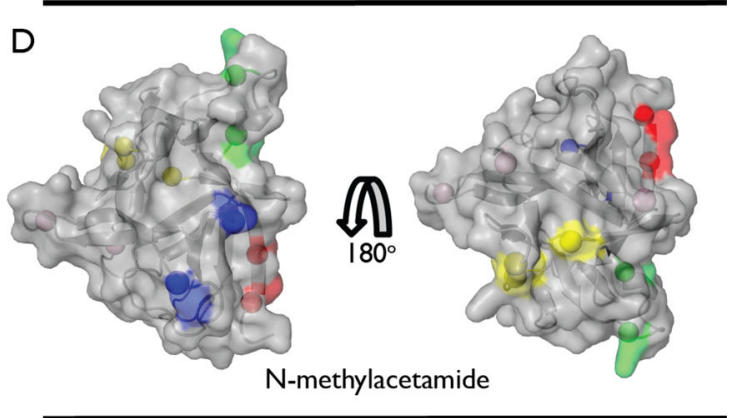

$\mathrm{F}$

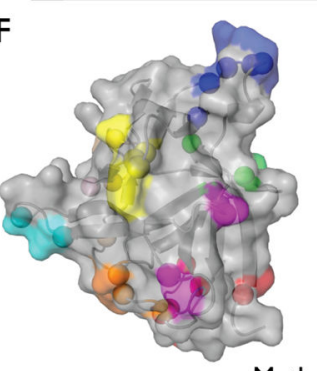

Methylammonium

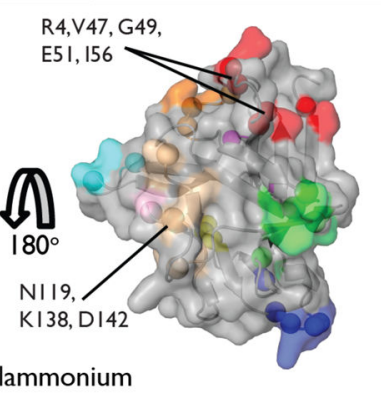

Results of cosolvent mapping experiments performed in reverse micelles. Clustering of residues into common binding sites was performed as described in the Supporting Information. Residues within a binding cluster are depicted in the same color. Binding affinities for each individual residue are included in Supplementary Table 2. Members of the tightest binding clusters $\left(\mathrm{K}_{\mathrm{d}}<1.25 \mathrm{M}\right)$ are labeled. Identities of clusters and their globally fitted $K_{d}$ values are given in Supplementary Table 3. Titrations were performed with (A) imidazole, (B) pyrimidine, (C) ethanol, (D) N-methylacetamide, (E) acetonitrile, and (F) 

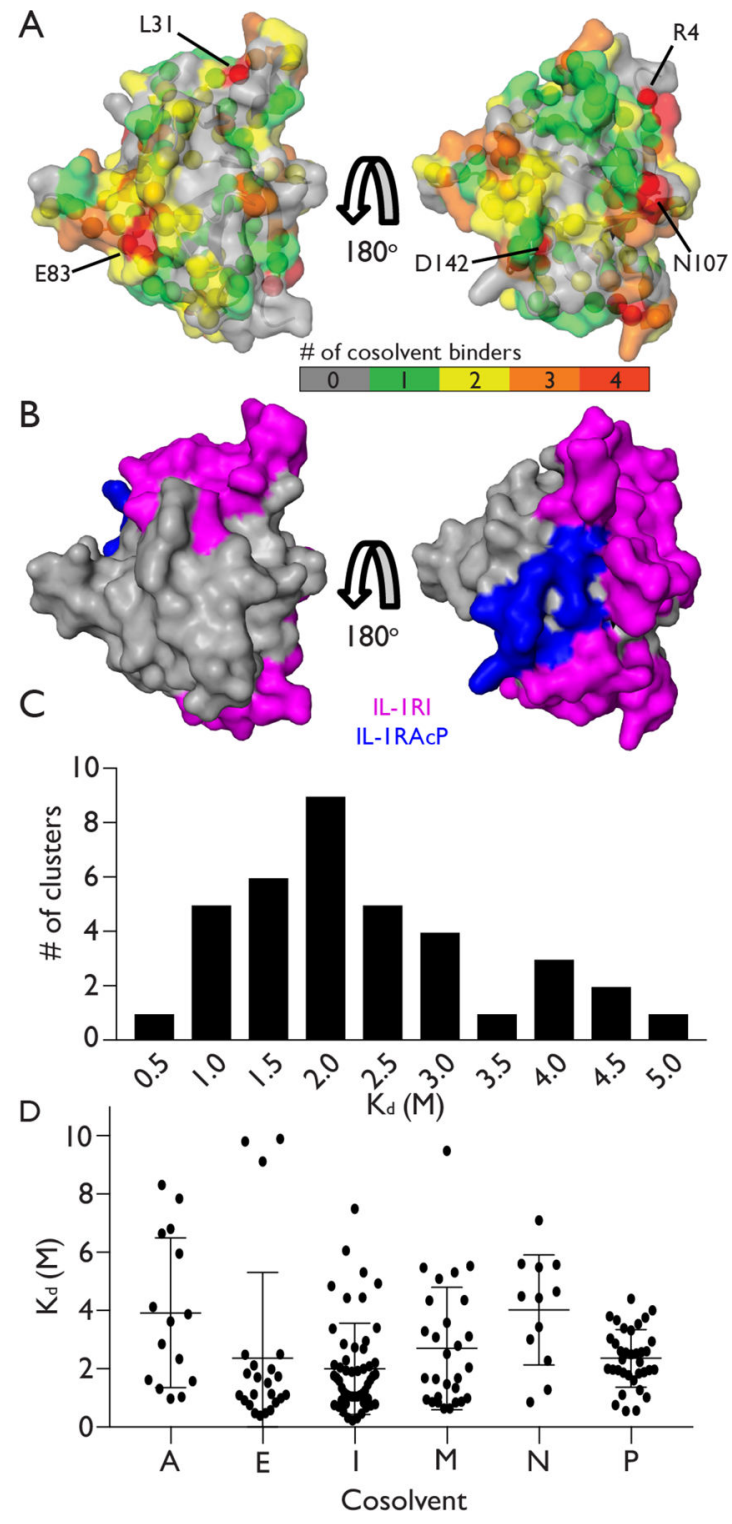

Figure 2.

Analysis of cosolvent hotspots and binding affinities. (A) Surface of IL-1 $\beta$ color-coded according to the number of cosolvents that generate a measureable CSP at each residue. Residues that bind 4 different cosolvents are labeled (B) Surface of IL-1 $\beta$ that binds to receptor proteins IL-1RI (magenta) and IL-1RAcP (blue). (C) Histogram of the binding affinities determined for all clusters containing two or more residues. (D) Dot-plot of binding affinities fit for all residues for each cosolvent: acetonitrile $(A)$, ethanol $(E)$, imidazole $(I)$, methylammonium chloride $(M)$, N-methylacetamide $(N)$, and pyrimidine $(P)$. Horizontal lines indicate the average and $+/-$ one standard deviation of the binding affinities determined for each cosolvent. 